\title{
An Adaptive Univariate Global Optimization Algorithm and Its Convergence Rate under the Wiener Measure
}

\author{
James CALVIN \\ Department of Computer Science, New Jersey Institute of Technology \\ Newark, NJ 07102-1982, USA \\ e-mail: calvin@njit.edu
}

Received: May 2011; accepted: October 2011

\begin{abstract}
We describe an adaptive algorithm for approximating the global minimum of a continuous univariate function. The convergence rate of the error is studied for the case of a random objective function distributed according to the Wiener measure.
\end{abstract}

Keywords: optimization, statistical models, convergence.

\section{Introduction}

Several algorithms have been proposed for global optimization that are motivated by a probability model for the objective function. A general Bayesian approach to optimization was formulated in Mockus (1972). For such algorithms it is interesting to analyze the convergence characteristics under the motivating stochastic model. A popular stochastic model is the Wiener process, due mainly to its tractability and also because it gives examples of very irregular multimodal functions which are interesting for global optimization. Optimization of nonsmooth objective functions arises in applications such as binary classification by support vector machines (Bartkute-Norkuniene, 2009). Optimization for the Wiener process model was considered in Kushner (1962). The algorithm described in this paper is of a form studied from the point of view of axiomatic rationality in Žilinskas (1985). The issue that we address is chosing the parameters of the algorithm so that the algorithm is efficient and also so that the convergence rate can be identified.

In this paper we propose an algorithm for approximating the global minimum of a continuous function $f$ defined on the unit interval. Under the assumption that $f$ is a random function distributed according to the Wiener measure, we establish an asymptotic upper bound on its approximation error.

Let $f$ be a Wiener process on $[0,1]$. That is, $f$ is a continuous Gaussian process with covariance function $E f(s) f(t)=\min (s, t), 0 \leqslant s, t \leqslant 1$. We are interested in approximating the global minimum $M \equiv \min _{0 \leqslant t \leqslant 1} f(t)$ of $f$ using a number of adaptively chosen function evaluations. The algorithm specifies a first point $t_{1}$ at which to evaluate $f\left(t_{1}\right)$. The algorithm then computes the second point $t_{2}=t_{2}\left(f\left(t_{1}\right)\right)$, and evaluates 
$f\left(t_{2}\right)$. Continuing on in this fashion, we evaluate

$$
f\left(t_{1}\right), f\left(t_{1}\right), \ldots, f\left(t_{n}\right)
$$

where $t_{k}=t_{k}\left(f\left(t_{1}\right), \ldots, f\left(t_{k-1}\right)\right)$ for $k \leqslant n$.

For notational convenience set $t_{0}=0$ and $t_{i}^{n}$ the $i$ th smallest of the $\left\{t_{j}\right\}$, so that $0=t_{0}^{n}<t_{1}^{n}<\cdots<t_{n}^{n} \leqslant 1$ and $\left\{t_{i}^{n}: 0 \leqslant i \leqslant n\right\}=\left\{t_{i}: 0 \leqslant i \leqslant n\right\}$. Let $\tau_{n}=$ $\min _{1 \leqslant i \leqslant n} t_{i}^{n}-t_{i-1}^{n}$ denote the smallest distance between function evaluation points.

The next section describes the details of the proposed algorithm and states the convergence theorem. The convergence analysis and proof of the theorem are in Section 3.

\section{The Algorithm}

The first two observation points of the algorithm are fixed: $t_{1}=1$ and $t_{2}=1 / 2$. Define

$$
g(x)=\sqrt{\lambda x \log (1 / x)}
$$

for $0<x \leqslant 1 / 2$. Here $\lambda$ is a fixed number $\geqslant 16$, which is convenient to leave unspecified. Note that $g$ is increasing and $g(x) \downarrow 0$ as $x \downarrow 0$. Set

$$
\rho_{i}^{n}=\frac{t_{i}^{n}-t_{i-1}^{n}}{\left(f\left(t_{i-1}^{n}\right)-M_{n}+g\left(\tau_{n}\right)\right)\left(f\left(t_{i}^{n}\right)-M_{n}+g\left(\tau_{n}\right)\right)} .
$$

The algorithm operates as follows: at each step, split the interval with the largest value of $\rho_{i}^{n} \equiv \rho^{n}$ at the midpoint. More precisely, suppose we have made $k$ evaluations. Compute $\rho_{i}^{k}, 1 \leqslant i \leqslant k$, and let $i$ be an index such that $\rho_{i}^{k} \geqslant \rho_{j}^{k}$ for all $1 \leqslant j \leqslant k$. The next function evaluation is made at the midpoint

$$
t_{k+1}=\frac{t_{i-1}^{k}+t_{i}^{k}}{2}
$$

Denote the error by $\Delta_{n}=\min _{1 \leqslant i \leqslant n} f\left(t_{i}\right)-\min _{0 \leqslant t \leqslant 1} f(t)$.

Our main result is

Theorem 1. For the algorithm described above, as the number of observations $n \rightarrow \infty$, there exists a positive constant $c$ such that

$$
P\left(\Delta_{n} \leqslant(c n)^{1 / 4} \exp \left(-\frac{1}{2} \sqrt{c n}\right)\right) \rightarrow 1 .
$$




\section{Convergence}

Suppose that the algorithm is about to split the smallest subinterval at time $n$; that is, $\rho^{n}=\rho_{i}^{n}$ and $\tau_{n}=t_{i}^{n}-t_{i-1}^{n}$. Then

$$
\rho^{n} \leqslant \frac{1}{\lambda \log \left(1 / \tau_{n}\right)} \leqslant \frac{1}{\lambda \log (n)}
$$

since $\tau_{n} \leqslant 1 / n$.

Consider the $2 n-1$ random variables

$$
\frac{f\left(t_{i}^{k}\right)-f\left(t_{i-1}^{k}\right)}{\sqrt{t_{i}^{k}-t_{i-1}^{k}}}, \quad 1 \leqslant i \leqslant k \leqslant n .
$$

These are standard normal random variables, with nonnegative covariances, since

$$
\operatorname{Cov}\left(\frac{f\left(t_{i}^{l}\right)-f\left(t_{i-1}^{l}\right)}{\sqrt{t_{i}^{l}-t_{i-1}^{l}}}, \frac{f\left(t_{j}^{m}\right)-f\left(t_{j-1}^{m}\right)}{\sqrt{t_{j}^{m}-t_{j-1}^{m}}}\right)=\left(\frac{\min \left(t_{i}^{l}, t_{j}^{m}\right)-\max \left(t_{i-1}^{l}, t_{j-1}^{m}\right)}{\max \left(t_{i}^{l}, t_{j}^{m}\right)-\min \left(t_{i-1}^{l}, t_{j-1}^{m}\right)}\right)^{1 / 2} .
$$

Therefore, by the Slepian-Schläfli form of the comparison principle for Gaussian random variables (Lifshits, 1995),

$$
P\left(\max _{1 \leqslant k \leqslant n} \max _{1 \leqslant i \leqslant k} \frac{f\left(t_{i}^{k}\right)-f\left(t_{i-1}^{k}\right)}{\sqrt{t_{i}^{k}-t_{i-1}^{k}}} \leqslant r\right) \geqslant P\left(\max _{1 \leqslant i \leqslant 2 n-1} U_{i} \leqslant r\right)
$$

for any $r \in \mathcal{R}$, where the $U_{i}$ are independent standard normal random variables.

Let

$$
W_{n}=\max _{1 \leqslant k \leqslant n} \max _{1 \leqslant i \leqslant k} \frac{\left|f\left(t_{i}^{k}\right)-f\left(t_{i-1}^{k}\right)\right|}{\sqrt{t_{i}^{k}-t_{i-1}^{k}}} .
$$

Then

$$
\begin{aligned}
P\left(W_{n} \leqslant 2 \sqrt{\log (n)}\right) & =(2 \Phi(2 \sqrt{\log (n)})-1)^{2 n-1} \\
& \geqslant\left(2-\frac{2}{\sqrt{2 \pi}} \frac{1}{2 \sqrt{\log (n)}} \exp \left(-\frac{1}{2} 4 \log (n)\right)-1\right)^{2 n-1} \\
& =\left(1-\frac{1}{2 n-1}\left(\frac{1}{\sqrt{2 \pi}} \frac{1}{\sqrt{\log (n)}} \frac{2 n-1}{n^{2}}\right)\right)^{2 n-1} \\
& \rightarrow 1
\end{aligned}
$$

where $\Phi$ is the normal cumulative distribution function and we used the inequality

$$
\Phi(x) \geqslant 1-\frac{1}{\sqrt{2 \pi}} \frac{1}{x} \exp \left(-x^{2} / 2\right)
$$


(Lifshits, 1995). Define

$$
F_{n}=\left\{f: \max _{1 \leqslant k \leqslant n} \max _{1 \leqslant i \leqslant k} \frac{\left|f\left(t_{i}^{k}\right)-f\left(t_{i-1}^{k}\right)\right|}{\sqrt{t_{i}^{k}-t_{i-1}^{k}}} \leqslant 2 \sqrt{\log (n)}\right\}
$$

Then $P\left(F_{n}\right) \rightarrow 1$.

Equation (1) gives an upper bound on $\rho^{n}$ at times when the algorithm is about to form a new smallest subinterval. The following lemma provides a high-probability upper bound at other times.

Lemma 1. For all $n \geqslant 2$ and $f \in F_{n}$,

$$
\rho^{n} \leqslant \frac{2}{\lambda \log \left(1 / \tau_{n}\right)} \leqslant \frac{2}{\lambda \log (n)} .
$$

Proof. The second inequality follows from the fact that $\tau_{n} \leqslant 1 / n$.

Let $m \leqslant n$ be the last time before $n$ that the smallest subinterval was about to be split, so $\tau_{m}=2 \tau_{n}$ and by (1),

$$
\rho^{m} \leqslant \frac{1}{\lambda \log \left(1 / \tau_{m}\right)}=\frac{1}{\lambda \log \left(1 / 2 \tau_{n}\right)} .
$$

We will show by induction that

$$
\rho^{m+k} \leqslant \frac{2}{\lambda \log \left(1 / \tau_{m+k}\right)}, \quad k=1,2, \ldots, n-m .
$$

Let us first consider the $\rho$ values for time $m+1$. Suppose that $i$ is the split interval at time $m$, and let $j$ denote a non-split subinterval, so $\rho_{j}^{m} \leqslant \rho^{m}$. Then

$$
\begin{aligned}
& \rho_{j}^{m+1}=\frac{t_{j}^{m}-t_{j-1}^{n}}{\left(f\left(t_{j-1}^{m}\right)-M_{m+1}+g\left(\tau_{n}\right)\right)\left(f\left(t_{j}^{m}\right)-M_{m+1}+g\left(\tau_{n}\right)\right)} \\
& \leqslant \frac{t_{j}^{m}-t_{j-1}^{n}}{\left(f\left(t_{j-1}^{m}\right)-M_{m}+g\left(\tau_{n}\right)\right)\left(f\left(t_{j}^{m}\right)-M_{m}+g\left(\tau_{n}\right)\right)} \\
& \leqslant\left(\frac{g\left(2 \tau_{n}\right)}{g\left(\tau_{n}\right)}\right)^{2} \frac{t_{j}^{m}-t_{j-1}^{n}}{\left(f\left(t_{j-1}^{n}\right)-M_{m}+g\left(2 \tau_{n}\right)\right)\left(f\left(t_{j}^{n}\right)-M_{m}+g\left(2 \tau_{n}\right)\right)} \\
& \text { since } g\left(2 \tau_{n}\right)>g\left(\tau_{n}\right) \\
& =\left(\frac{g\left(2 \tau_{n}\right)}{g\left(\tau_{n}\right)}\right)^{2} \rho_{j}^{m} \\
& =\frac{\lambda 2 \tau_{n} \log \left(1 / 2 \tau_{n}\right)}{\lambda \tau_{n} \log \left(1 / \tau_{n}\right)} \rho_{j}^{m} \\
& =2 \frac{\log \left(1 / 2 \tau_{n}\right)}{\log \left(1 / \tau_{n}\right)} \rho_{j}^{m}
\end{aligned}
$$




$$
\begin{aligned}
& \leqslant 2 \frac{\log \left(1 / 2 \tau_{n}\right)}{\log \left(1 / \tau_{n}\right)} \rho^{m} \\
& \leqslant 2 \frac{\log \left(1 / 2 \tau_{n}\right)}{\log \left(1 / \tau_{n}\right)} \frac{1}{\lambda \log \left(1 / \tau_{m}\right)} \quad \text { by }(1) \\
& =2 \frac{\log \left(1 / 2 \tau_{n}\right)}{\log \left(1 / \tau_{n}\right)} \frac{1}{\lambda \log \left(1 / 2 \tau_{n}\right)} \\
& =\frac{2}{\lambda \log \left(1 / \tau_{n}\right)} \\
& =\frac{2}{\lambda \log \left(1 / \tau_{m+1}\right)}
\end{aligned}
$$

Next consider a child of the split subinterval $i$. Since we are splitting the smallest subinterval, $t_{i}^{m}-t_{i-1}^{m}=\tau_{m}=2 \tau_{n}$, and

$$
\begin{aligned}
\rho_{i}^{m+1} & =\frac{\tau_{n}}{\left(f\left(t_{i-1}^{m}\right)-M_{m+1}+g\left(\tau_{n}\right)\right)\left(f\left(t_{m+1}\right)-M_{m+1}+g\left(\tau_{n}\right)\right)} \\
& \leqslant \frac{\tau_{n}}{g\left(\tau_{n}\right)^{2}} \\
& =\frac{1}{\lambda \log \left(1 / \tau_{n}\right)} \\
& =\frac{1}{\lambda \log \left(1 / \tau_{m+1}\right)} .
\end{aligned}
$$

We have established the base case for the induction. Now let's consider iteration $m+$ $k+1,1 \leqslant k<n-m$. For the induction hypothesis, assume that

$$
\rho^{m+k} \leqslant \frac{2}{\lambda \log \left(1 / \tau_{m+k}\right)}=\frac{2}{\lambda \log \left(1 / \tau_{n}\right)}
$$

Suppose we evaluate at $t_{m+k+1}=\left(t_{i-1}^{m+k}+t_{i}^{m+k}\right) / 2$. Then

$$
f\left(t_{m+k+1}\right)=\frac{f\left(t_{i-1}^{m+k}\right)+f\left(t_{i}^{m+k}\right)}{2}+\delta
$$

where $f \in F_{n}$ implies that (let $T_{i}=t_{i}^{m+k}-t_{i-1}^{m+k}$ )

$$
\left|\frac{f\left(t_{i-1}^{m+k}\right)+f\left(t_{i}^{m+k}\right)}{2}+\delta-f\left(t_{i-1}^{m+k}\right)\right| \leqslant \sqrt{T_{i} / 2} \cdot 2 \sqrt{\log (n)}
$$

and

$$
\left|\frac{f\left(t_{i-1}^{m+k}\right)+f\left(t_{i}^{m+k}\right)}{2}+\delta-f\left(t_{i}\right)\right| \leqslant \sqrt{T_{i} / 2} \cdot 2 \sqrt{\log (n)} .
$$


Equivalently,

$$
\left|-\frac{f\left(t_{i-1}^{m+k}\right)-f\left(t_{i}^{m+k}\right)}{2}+\delta\right| \leqslant \sqrt{2 T_{i} \log (n)}
$$

and

$$
\left|\frac{f\left(t_{i-1}^{m+k}\right)-f\left(t_{i}^{m+k}\right)}{2}+\delta\right| \leqslant \sqrt{2 T_{i} \log (n)} .
$$

Therefore,

$$
\begin{aligned}
\delta & \leqslant-\frac{\left|f\left(t_{i-1}^{m+k}\right)-f\left(t_{i}^{m+k}\right)\right|}{2}+\sqrt{2 T_{i} \log (n)} \\
& \leqslant-\frac{\left|f\left(t_{i-1}^{m+k}\right)-f\left(t_{i}^{m+k}\right)\right|}{2}+\sqrt{2 T_{i} \log \left(1 / \tau_{n}\right)} .
\end{aligned}
$$

Then (considering without loss of generality the left child)

$$
\begin{aligned}
\frac{\rho_{i}^{m+k+1}}{\rho_{i}^{m+k}}= & \frac{1}{2} \frac{\left(f\left(t_{i-1}^{m+k}\right)-M_{m+k}+g\left(\tau_{n}\right)\right)\left(f\left(t_{i}^{m+k}\right)-M_{m+k}+g\left(\tau_{n}\right)\right)}{\left(f\left(t_{i-1}^{m+k}\right)-M_{m+k+1}+g\left(\tau_{n}\right)\right)\left(f\left(t_{m+k+1}\right)-M_{m+k+1}+g\left(\tau_{n}\right)\right)} \\
\leqslant & \frac{1}{2} \frac{\left(f\left(t_{i-1}^{m+k}\right)-M_{m+k}+g\left(\tau_{n}\right)\right)\left(f\left(t_{i}^{m+k}\right)-M_{m+k}+g\left(\tau_{n}\right)\right)}{\left(f\left(t_{i-1}^{m+k}\right)-M_{m+k}+g\left(\tau_{n}\right)\right)\left(f\left(t_{m+k+1}\right)-M_{m+k+1}+g\left(\tau_{n}\right)\right)} \\
= & \frac{1}{2} \frac{\left(f\left(t_{i}^{m+k}\right)-M_{m+k}+g\left(\tau_{n}\right)\right)}{\left(f\left(t_{m+k+1}\right)-M_{m+k+1}+g\left(\tau_{n}\right)\right)} \\
\leqslant & {\left[f\left(t_{i}^{m+k}\right)-M_{m+k}+g\left(\tau_{n}\right)\right]\left[f\left(t_{i-1}^{m+k}\right)-M_{m+k}+g\left(\tau_{n}\right)+f\left(t_{i}^{m+k}\right)\right.} \\
& \left.-M_{m+k}+g\left(\tau_{n}\right)+\left|f\left(t_{i-1}^{m+k}\right)-f\left(t_{i}^{m+k}\right)\right| / 2-\sqrt{2 T_{i} \log \left(1 / \tau_{n}\right)}\right]^{-1} .
\end{aligned}
$$

Making the substitution

$$
x \equiv \frac{\sqrt{f\left(t_{i}^{m+k}\right)-M_{m+k}+g\left(\tau_{n}\right)}}{\sqrt{f\left(t_{i-1}^{m+k}\right)-M_{m+k}+g\left(\tau_{n}\right)}}>0,
$$

the last expression is

$$
\begin{aligned}
\frac{\rho_{i}^{m+k+1}}{\rho_{i}^{m+k}} & \leqslant \frac{x}{x+\frac{1}{x}+\frac{1}{2}\left|x-\frac{1}{x}\right|-\sqrt{\rho^{m+k}} \sqrt{2 \log \left(1 / \tau_{n}\right)}} \\
& \leqslant \frac{x}{x+\frac{1}{x}+\frac{1}{2}\left|x-\frac{1}{x}\right|-\frac{2}{\sqrt{\lambda}}} \leqslant 1
\end{aligned}
$$

by the induction hypothesis if $\lambda \geqslant 4$. The proof by induction of (2) is complete. 
Let $N_{n}(f)$ denote the $\sigma$-algebra generated by $\left(f\left(t_{1}\right), f\left(t_{2}\right), \ldots, f\left(t_{n}\right)\right)$.

Lemma 2. If $1 \geqslant \beta>\frac{1}{\sqrt{2 \lambda}} \approx 0.707 / \sqrt{\lambda}$, then for all $f \in F_{n}$,

$$
P\left(\Delta_{n} \leqslant \beta g\left(\tau_{n}\right) \mid N_{n}(f)\right) \geqslant 1-n^{1-2 \beta^{2} \lambda} .
$$

Note that the lower bound converges to 1 as $n \rightarrow \infty$ by our assumption on $\beta$.

Proof. Conditional on $N_{n}(f)$, the minimizers over distinct subintervals $\left[t_{i-1}^{n}, t_{i}^{n}\right]$ and $\left[t_{j-1}^{n}, t_{j}^{n}\right]$ are independent with distribution

$$
P\left(\min _{t_{i-1}^{n} \leqslant s \leqslant t_{i}^{n}} f(s)<y\right)=\exp \left(-\frac{2}{t_{i}^{n}-t_{i-1}^{n}}\left(f\left(t_{i-1}^{n}\right)-y\right)\left(f\left(t_{i}^{n}\right)-y\right)\right)
$$

for $y<\min \left(f\left(t_{i-1}^{n}\right), f\left(t_{i}^{n}\right)\right)$; see (Shepp, 1979). Therefore,

$$
\begin{aligned}
P\left(\Delta_{n} \leqslant\right. & \left.\beta g\left(\tau_{n}\right) \mid N_{n}(f)\right) \\
= & \prod_{i=1}^{n}\left(1-\exp \left(-\frac{2}{t_{i}^{n}-t_{i-1}^{n}}\left(f\left(t_{i-1}^{n}\right)-M_{n}+\beta g\left(\tau_{n}\right)\right)\right.\right. \\
& \left.\left.\times\left(f\left(t_{i}^{n}\right)-M_{n}+\beta g\left(\tau_{n}\right)\right)\right)\right) \\
\geqslant & \prod_{i=1}^{n}\left(1-\exp \left(-\frac{2 \beta^{2}}{t_{i}^{n}-t_{i-1}^{n}}\left(f\left(t_{i-1}^{n}\right)-M_{n}+g\left(\tau_{n}\right)\right)\right.\right. \\
& \left.\left.\times\left(f\left(t_{i}^{n}\right)-M_{n}+g\left(\tau_{n}\right)\right)\right)\right) \\
= & \prod_{i=1}^{n}\left(1-\exp \left(-\frac{2 \beta^{2}}{\rho_{i}^{n}}\right)\right) \\
\geqslant & \left(1-\exp \left(-\frac{2 \beta^{2}}{\rho^{n}}\right)\right)^{n} \\
\geqslant & \left(1-\exp \left(-2 \beta^{2} \lambda \log (n)\right)\right)^{n} \\
= & \left(1-\frac{n^{1-2 \beta^{2} \lambda}}{n}\right)^{n} \\
\geqslant & 1-n^{1-2 \beta^{2} \lambda} .
\end{aligned}
$$

Define the linear interpolation $L_{n}$ of the $\left\{f\left(t_{i}^{n}\right)\right\}$ by

$$
\begin{aligned}
L_{n}(s) & =\frac{t_{i}^{n}-s}{t_{i}^{n}-t_{i-1}^{n}} f\left(t_{i-1}^{n}\right)+\frac{s-t_{i-1}^{n}}{t_{i}^{n}-t_{i-1}^{n}} f\left(t_{i}^{n}\right), \\
t_{i-1}^{n} & \leqslant s \leqslant t_{i}^{n}, 1 \leqslant i \leqslant n .
\end{aligned}
$$


Then

$$
\rho_{i}^{n}=\int_{s=t_{i-1}^{n}}^{t_{i}^{n}} \frac{d s}{\left(L_{n}(s)-M_{n}+g\left(\tau_{n}\right)\right)^{2}}
$$

\section{Lemma 3.}

$$
\begin{aligned}
& \lim _{n \rightarrow \infty} P\left(\int_{t=0}^{1} \frac{d t}{\left(L_{n}(t)-M+g\left(\tau_{n}\right)\right)^{2}}\right. \\
& \left.\leqslant \sum_{i=1}^{n} \rho_{i}^{n} \leqslant\left(1-\frac{3}{4 \sqrt{\lambda}}\right)^{-2} \int_{t=0}^{1} \frac{d t}{\left(L_{n}(t)-M+g\left(\tau_{n}\right)\right)^{2}}\right)=1
\end{aligned}
$$

Proof. The first inequality follows from

$$
\sum_{i=1}^{n} \rho_{i}^{n}=\int_{t=0}^{1} \frac{d t}{\left(L_{n}(t)-M_{n}+g\left(\tau_{n}\right)\right)^{2}} \geqslant \int_{t=0}^{1} \frac{d t}{\left(L_{n}(t)-M+g\left(\tau_{n}\right)\right)^{2}}
$$

For the second inequality,

$$
\sum_{i=1}^{n} \rho_{i}^{n}=\int_{t=0}^{1} \frac{d t}{\left(L_{n}(t)-M_{n}+g\left(\tau_{n}\right)\right)^{2}}=\int_{t=0}^{1} \frac{d t}{\left(L_{n}(t)-M+g\left(\tau_{n}\right)-\Delta_{n}\right)^{2}}
$$

Set

$$
A_{n}=\left\{f: \Delta_{n}(f) \leqslant \frac{3}{4 \sqrt{\lambda}} g\left(\tau_{n}\right)\right\}
$$

By Lemma $2, P\left(A_{n}\right) \rightarrow 1$. On $A_{n}$,

$$
\begin{aligned}
& \int_{t=0}^{1} \frac{d t}{\left(L_{n}(t)-M+g\left(\tau_{n}\right)-\Delta_{n}\right)^{2}} \\
& \quad \leqslant \int_{t=0}^{1} \frac{d t}{\left(L_{n}(t)-M+\left(1-\frac{3}{4 \sqrt{\lambda}}\right) g\left(\tau_{n}\right)\right)^{2}} \\
& \quad \leqslant\left(1-\frac{3}{4 \sqrt{\lambda}}\right)^{-2} \int_{t=0}^{1} \frac{d t}{\left(L_{n}(t)-M+g\left(\tau_{n}\right)\right)^{2}} .
\end{aligned}
$$


Lemma 4. Define the events

$$
\begin{aligned}
& B_{n}^{+}=\left\{\max _{1 \leqslant i \leqslant n} \max _{t_{i-1}^{n} \leqslant s \leqslant t_{i}^{n}} \frac{f(s)-L_{n}(s)}{\left.\sqrt{t_{i}^{n}-t_{i-1}^{n}} \leqslant \sqrt{\log (n)}\right\},}\right. \\
& B_{n}^{-}=\left\{\min _{1 \leqslant i \leqslant n} \min _{t_{i-1}^{n} \leqslant s \leqslant t_{i}^{n}} \frac{f(s)-L_{n}(s)}{\sqrt{t_{i}^{n}-t_{i-1}^{n}}} \geqslant-\sqrt{\log (n)}\right\} .
\end{aligned}
$$

Then $P\left(B_{n}^{+}\right), P\left(B_{n}^{-}\right) \rightarrow 1$ as $n \rightarrow \infty$.

Proof. Let

$$
Y_{i} \equiv \max _{t_{i-1}^{n} \leqslant s \leqslant t_{i}^{n}} \frac{f(s)-L_{n}(s)}{\sqrt{t_{i}^{n}-t_{i-1}^{n}}},
$$

which is the maximum of a standard Brownian bridge. Then $P\left(Y_{i}>y\right)=\exp \left(-2 y^{2}\right)$, $y \geqslant 0$; see (Shepp, 1979) and since the $Y_{i}$ 's are independent,

$$
P\left(\max _{1 \leqslant i \leqslant n} Y_{i} \leqslant \sqrt{\log (n)}\right)=(1-\exp (-2 \log (n)))^{n}=\left(1-\frac{1}{n^{2}}\right)^{n} \rightarrow 1 .
$$

Lemma 5. For $f \in F_{n}$,

$$
\begin{aligned}
& \max _{1 \leqslant i \leqslant n} \frac{t_{i}^{n}-t_{i-1}^{n}}{\left(\min \left\{f\left(t_{i-1}^{n}\right), f\left(t_{i}^{n}\right)\right\}-M+g\left(\tau_{n}\right)\right)^{2}} \\
& \quad \leqslant \max _{1 \leqslant i \leqslant n} \frac{t_{i}^{n}-t_{i-1}^{n}}{\left(\min \left\{f\left(t_{i-1}^{n}\right), f\left(t_{i}^{n}\right)\right\}-M_{n}+g\left(\tau_{n}\right)\right)^{2}} \leqslant \frac{A(\lambda)}{\log (n)},
\end{aligned}
$$

where

$$
A(\lambda)=\left(\frac{1}{\lambda}+\sqrt{\frac{1}{\lambda^{2}}+\frac{2}{\lambda}}\right)^{2}
$$

Proof. Since $M_{n} \geqslant M$, we need only prove the second inequality. Observe that

$$
\begin{aligned}
\max _{1 \leqslant i \leqslant n} & \frac{t_{i}^{n}-t_{i-1}^{n}}{\left(\min \left\{f\left(t_{i-1}^{n}\right), f\left(t_{i}^{n}\right)\right\}-M_{n}+g\left(\tau_{n}\right)\right)^{2}} \\
= & \max _{1 \leqslant i \leqslant n} \frac{t_{i}^{n}-t_{i-1}^{n}}{\left(f\left(t_{i-1}^{n}\right)-M_{n}+g\left(\tau_{n}\right)\right)\left(f\left(t_{i}^{n}\right)-M_{n}+g\left(\tau_{n}\right)\right)} \\
& \times\left(1+\frac{\left|f\left(t_{i}^{n}\right)-f\left(t_{i-1}^{n}\right)\right|}{\min \left\{f\left(t_{i-1}^{n}\right), f\left(t_{i}^{n}\right)\right\}-M_{n}+g\left(\tau_{n}\right)}\right) \\
\leqslant & \rho^{n}\left(1+\max _{1 \leqslant i \leqslant n} \frac{\left|f\left(t_{i}^{n}\right)-f\left(t_{i-1}^{n}\right)\right|}{\min \left\{f\left(t_{i-1}^{n}\right), f\left(t_{i}^{n}\right)\right\}-M_{n}+g\left(\tau_{n}\right)}\right)
\end{aligned}
$$




$$
\begin{aligned}
= & \rho^{n}\left(1+\max _{1 \leqslant i \leqslant n} \frac{\left|f\left(t_{i}^{n}\right)-f\left(t_{i-1}^{n}\right)\right|}{\sqrt{t_{i}^{n}-t_{i-1}^{n}}} \frac{\sqrt{t_{i}^{n}-t_{i-1}^{n}}}{\min \left\{f\left(t_{i-1}^{n}\right), f\left(t_{i}^{n}\right)\right\}-M_{n}+g\left(\tau_{n}\right)}\right) \\
\leqslant & \rho^{n}\left(1+\max _{1 \leqslant i \leqslant n} \frac{\left|f\left(t_{i}^{n}\right)-f\left(t_{i-1}^{n}\right)\right|}{\sqrt{t_{i}^{n}-t_{i-1}^{n}}}\right. \\
& \left.\times \max _{1 \leqslant i \leqslant n} \frac{\sqrt{t_{i}^{n}-t_{i-1}^{n}}}{\min \left\{f\left(t_{i-1}^{n}\right), f\left(t_{i}^{n}\right)\right\}-M_{n}+g\left(\tau_{n}\right)}\right) .
\end{aligned}
$$

Setting

$$
z_{n} \equiv \max _{1 \leqslant i \leqslant n} \frac{\sqrt{t_{i}^{n}-t_{i-1}^{n}}}{\min \left\{f\left(t_{i-1}^{n}\right), f\left(t_{i}^{n}\right)\right\}-M_{n}+g\left(\tau_{n}\right)},
$$

the last inequality reads

$$
z_{n}^{2} \leqslant \rho^{n}\left(1+\max _{1 \leqslant i \leqslant n} \frac{\left|f\left(t_{i}^{n}\right)-f\left(t_{i-1}^{n}\right)\right|}{\sqrt{t_{i}^{n}-t_{i-1}^{n}}} \cdot z_{n}\right) .
$$

Now use the fact that $f \in F_{n}$ and $\rho^{n} \leqslant 2 / \lambda \log (n)$ (from Lemma 2) to get

$$
z_{n}^{2} \leqslant \frac{2}{\lambda \log (n)}\left(1+2 \sqrt{\log (n)} \cdot z_{n}\right)
$$

or

$$
\left(z_{n}-\frac{1}{\lambda \sqrt{\log (n)}}\right)^{2} \leqslant \frac{1}{\lambda^{2} \log (n)}+\frac{2}{\lambda \log (n)} .
$$

This implies that

$$
z_{n} \leqslant \frac{1}{\sqrt{\log (n)}}\left(\frac{1}{\lambda}+\sqrt{\frac{1}{\lambda^{2}}+\frac{2}{\lambda}}\right),
$$

which is the desired inequality.

\section{Lemma 6.}

$$
\begin{gathered}
\lim _{n \rightarrow \infty} P\left(\frac{1}{4} \int_{t=0}^{1} \frac{d t}{\left(f(t)-M+g\left(\tau_{n}\right)\right)^{2}} \leqslant \sum_{i=1}^{n} \rho_{i}^{n}\right. \\
\left.\leqslant \frac{9}{4} \int_{t=0}^{1} \frac{d t}{\left(f(t)-M+g\left(\tau_{n}\right)\right)^{2}}\right)=1 .
\end{gathered}
$$


Proof.

$$
\begin{aligned}
& \int_{t=0}^{1} \frac{d t}{\left(f(t)-M+g\left(\tau_{n}\right)\right)^{2}} \\
& \quad=\sum_{i=1}^{n} \int_{s=t_{i-1}}^{t_{i}} \frac{d s}{\left(L_{n}(s)-M+g\left(\tau_{n}\right)\right)^{2}\left(1+\frac{f(s)-L_{n}(s)}{L_{n}(s)-M+g\left(\tau_{n}\right)}\right)^{2}} \\
& \quad=\sum_{i=1}^{n} \int_{s=t_{i-1}}^{t_{i}} \frac{d s}{\left(L_{n}(s)-M+g\left(\tau_{n}\right)\right)^{2}\left(1+\frac{f(s)-L_{n}(s)}{\sqrt{t_{i}^{n}-t_{i-1}^{n}}} \frac{\sqrt{t_{i}^{n}-t_{i-1}^{n}}}{L_{n}(s)-M+g\left(\tau_{n}\right)}\right)^{2}} .
\end{aligned}
$$

On the event $F_{n} \cap B_{n}^{+}$, using Lemma 5,

$$
\begin{aligned}
& \frac{f(s)-L_{n}(s)}{\sqrt{t_{i}^{n}-t_{i-1}^{n}}} \frac{\sqrt{t_{i}^{n}-t_{i-1}^{n}}}{L_{n}(s)-M+g\left(\tau_{n}\right)} \\
& \quad \leqslant \frac{f(s)-L_{n}(s)}{\sqrt{t_{i}^{n}-t_{i-1}^{n}}} \frac{\sqrt{t_{i}^{n}-t_{i-1}^{n}}}{\min \left(f\left(t_{i-1}^{n}\right), f\left(t_{i}^{n}\right)\right)-M+g\left(\tau_{n}\right)} \\
& \quad \leqslant \sqrt{\log (n)} \cdot \frac{A(\lambda)^{1 / 2}}{\sqrt{\log (n)}} \\
& \quad=\left(\frac{1}{\lambda}+\sqrt{\frac{1}{\lambda^{2}}+\frac{2}{\lambda}}\right) \leqslant 1 / 2
\end{aligned}
$$

if $\lambda \geqslant 16$. Similarly, on the event $F_{n} \cap B_{n}^{-}$,

$$
\frac{f(s)-L_{n}(s)}{\sqrt{t_{i}^{n}-t_{i-1}^{n}}} \frac{\sqrt{t_{i}^{n}-t_{i-1}^{n}}}{L_{n}(s)-M+g\left(\tau_{n}\right)} \geqslant-1 / 2
$$

if $\lambda \geqslant 16$. Therefore, on $F_{n} \cap B_{n}^{-} \cap B_{n}^{+}$,

$$
\int_{t=0}^{1} \frac{d t}{\left(f(t)-M+g\left(\tau_{n}\right)\right)^{2}} \leqslant 4 \sum_{i=1}^{n} \rho_{i}^{n}
$$

and

$$
\int_{t=0}^{1} \frac{d t}{\left(f(t)-M+g\left(\tau_{n}\right)\right)^{2}} \geqslant \frac{4}{9} \sum_{i=1}^{n} \rho_{i}^{n}
$$

if $\lambda \geqslant 16$.

Having established bounds for $\sum \rho_{i}^{n}$ in terms of $\int\left(f(t)-M+g\left(\tau_{n}\right)\right)^{-2} d t$, we next turn to estimating the growth of the integrals as $\tau_{n} \downarrow 0$. 
Lemma 7. As $n \rightarrow \infty$,

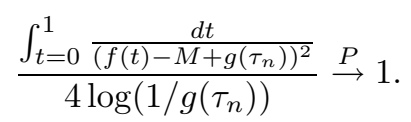

Proof. Let $t^{*}$ denote the (first) global minimizer of $f$. Conditional on $\left(t^{*}, f\left(t^{*}\right), f(1)\right)$, the process $\left(f\left(t^{*}+s\right)-f\left(t^{*}\right): 0 \leqslant s \leqslant 1-t^{*}\right)$ is a 3 -dimensional Bessel bridge over $\left[0,1-t^{*}\right]$ with terminal value $f(1)-f\left(t^{*}\right)$. Let $Y_{b}$ be a 3 -dimensional Bessel bridge over the interval $[0, t]$ with terminal value $Y_{b}(t)=b$, defined on a complete probability space $\left(\Omega, \mathcal{F}, P_{b}\right)$, and let $\mathcal{F}_{s} \equiv \sigma\left\{Y_{b}(u), u \leqslant s\right\}$ be the $\sigma$-algebra generated by the process up to time $s, 0 \leqslant s \leqslant t$. To prove the theorem it suffices to show that

$$
\frac{\int_{s=0}^{t} \frac{d s}{\left(Y_{b}(s)+\epsilon\right)^{2}}}{2 \log (1 / \epsilon)} \stackrel{P}{\rightarrow} 1
$$

as $\epsilon \downarrow 0$ (the factor of 2 in the denominator is because we are only considering the integral to the right of $t^{*}$; the integral to the left gives a similar contribution).

Define the event

$$
E_{b} \equiv\left\{\frac{\int_{s=0}^{t} \frac{d s}{\left(Y_{b}(s)+\epsilon\right)^{2}}}{2 \log (1 / \epsilon)} \rightarrow 1\right\}
$$

The event $E_{b}$ is measurable with respect to the $\sigma$-algebra

$$
\mathcal{F}_{0+}=\bigcap_{s>0} \mathcal{F}_{s}
$$

Since $Y_{b}$ is a Markov process, the Blumenthal $0-1$ law implies that $P_{b}\left(E_{b}\right) \in\{0,1\}$. We will show that $P_{b}\left(E_{b}\right)=1$, and since convergence with probability 1 implies convergence in probability, this will complete the proof.

Let $Y$ be an (unconditioned) 3-dimensional Bessel process, and set $\bar{Y}(s) \equiv$ $\min (1, Y(s))$ and let $L=\sup \{s: Y(s) \leqslant 1\}$ denote the last exit time of $Y$ from $[0,1]$. Now

$$
\int_{s=0}^{t} \frac{1_{\{Y(s) \leqslant 1\}} d s}{(Y(s)+\epsilon)^{2}}-\int_{s=0}^{t} \frac{d s}{(Y(s)+\epsilon)^{2}} \leqslant t /(1+\epsilon)^{2} \leqslant t
$$

and

$$
\int_{s=0}^{L} \frac{1_{\{Y(s) \leqslant 1\}} d s}{(Y(s)+\epsilon)^{2}}-\int_{s=0}^{t} \frac{1_{\{Y(s) \leqslant 1\}} d s}{(Y(s)+\epsilon)^{2}} \leqslant \frac{L-t}{V+\epsilon},
$$


where $V=\min _{t \leqslant u} Y(u)$, and so

$$
\frac{\int_{s=0}^{t} \frac{d s}{(Y(s)+\epsilon)^{2}}}{2 \log (1 / \epsilon)} \rightarrow 1
$$

if and only if

$$
\frac{\int_{s=0}^{\infty} \frac{1_{\{Y(s) \leqslant 1\} d s}}{(Y(s)+\epsilon)^{2}}}{2 \log (1 / \epsilon)} \rightarrow 1
$$

The local time (occupation density) of the 3 dimensional Bessel process is the square of a 2-dimensional Bessel process which has the same distribution as the squared-modulus of a 2-dimensional Wiener process. Therefore we can write

$$
\int_{s=0}^{\infty} \frac{1_{\{Y(s) \leqslant 1\}} d s}{(Y(s)+\epsilon)^{2}}=\int_{y=0}^{1} \frac{\left[B_{1}(y)^{2}+B_{2}(y)^{2}\right] d y}{(y+\epsilon)^{2}}
$$

and it suffices to show that

$$
\frac{\int_{y=0}^{1} \frac{B(y)^{2} d y}{(y+\epsilon)^{2}}}{\log (1 / \epsilon)} \rightarrow 1
$$

with probability 1 , where $B$ is a Wiener process.

Let us consider the process $Z(t)=F(B(t), t)$, where $F(x, t)=-x^{2}(1+t)^{-1}$. By Ito's formula,

$$
Z(t)=Z(0)+\int_{s=0}^{t} \frac{B(s)^{2}}{(1+s)^{2}} d s-2 \int_{s=0}^{t} \frac{B(s)}{1+s} d B(s)-\int_{s=0}^{t} \frac{d s}{1+s}
$$

or

$$
\begin{aligned}
\int_{s=0}^{t} \frac{B(s)^{2}}{(1+s)^{2}} d s & =-\frac{B(t)^{2}}{1+t}+\int_{s=0}^{t} \frac{d s}{1+s}+2 \int_{s=0}^{t} \frac{B(s) d B(s)}{1+s} \\
& =\log (1+t)-\frac{B(t)^{2}}{1+t}+2 \int_{s=0}^{t} \frac{B(s) d B(s)}{1+s}
\end{aligned}
$$

Therefore,

$$
\frac{\int_{t=0}^{1} \frac{B(t)^{2}}{(t+\epsilon)^{2}} d t}{\log (1 / \epsilon)}=\frac{\log (1+1 / \epsilon)-\frac{B(1 / \epsilon)^{2}}{1+1 / \epsilon}+2 \int_{s=0}^{1 / \epsilon} \frac{B(s) d B(s)}{1+s}}{\log (1 / \epsilon)}
$$




$$
=1+o(1)-\frac{B(1 / \epsilon)^{2}}{\log (1 / \epsilon)(1+1 / \epsilon)}+\frac{2}{\log (1 / \epsilon)} \int_{s=0}^{1 / \epsilon} \frac{B(s) d B(s)}{1+s} .
$$

The third term converges almost surely to 0 by the law of the iterated logarithm. The fourth term is an $L^{2}$-bounded martingale, since

$$
\begin{aligned}
& E\left(\frac{2}{\log (1 / \epsilon)} \int_{s=0}^{1 / \epsilon} \frac{B(s) d B(s)}{1+s}\right)^{2}=\left(\frac{2}{\log (1 / \epsilon)}\right)^{2} \int_{s=0}^{1 / \epsilon} E\left(\frac{B(s)}{1+s}\right)^{2} d s \\
& \quad=\left(\frac{2}{\log (1 / \epsilon)}\right)^{2} \int_{s=0}^{1 / \epsilon} \frac{s}{(1+s)^{2}} d s \\
& \quad=\left(\frac{2}{\log (1 / \epsilon)}\right)^{2}\left(\log (1+1 / \epsilon)+\frac{1}{1+1 / \epsilon}-1\right) \rightarrow 0
\end{aligned}
$$

as $\epsilon \rightarrow 0$. Therefore the fourth term is an $L^{2}$-bounded martingale, and so converges almost surely (to 0 ).

We have now shown that

$$
\frac{\int_{y=0}^{1} \frac{B_{1}(y)^{2} d y}{(y+\epsilon)^{2}}}{\log (1 / \epsilon)} \rightarrow 1
$$

almost surely, and so $P(E)=1$.

Finally, $1=P(E)=E P(E \mid Y(t))$, and so $P(E \mid Y(t)=y)=1$ for almost all $y>0$. By monotonicity, $P(E \mid Y(t)=y)=1$ for all $y>0$, and so $P\left(E_{b}\right)=1$.

\section{Lemma 8. Let}

$$
G_{n}=\left\{f: \rho^{k} \geqslant \frac{1}{\log \left(1 / \tau_{n}\right)(\lambda+2 \sqrt{\lambda})} \text { for } 1 \leqslant k \leqslant n\right\} .
$$

Then $F_{n} \subset G_{n}$; in particular, $P\left(G_{n}\right) \rightarrow 1$.

Proof. Suppose $k \leqslant n$ and $f \in F_{n}$. Let $i$ be an index such that $f\left(t_{i}^{k}\right)=M_{k}$, and observe that (letting $T_{i}=t_{i}^{k}-t_{i-1}^{k}$ )

$$
\begin{aligned}
\rho^{k} & \geqslant \rho_{i}^{k} \\
& =\frac{t_{i}^{k}-t_{i-1}^{k}}{g\left(\tau_{k}\right)\left(f\left(t_{i-1}^{k}\right)-M_{k}+g\left(\tau_{k}\right)\right)} \\
& =\frac{1}{\sqrt{\lambda\left(\tau_{k} / T_{i}\right) \log \left(1 / \tau_{k}\right)}\left(\left|f\left(t_{i}^{k}\right)-f\left(t_{i-1}^{k}\right)\right| / \sqrt{T_{i}}+\sqrt{\lambda\left(\tau_{k} / T_{i}\right) \log \left(1 / \tau_{k}\right)}\right)} \\
& \geqslant \frac{1}{\sqrt{\lambda \log \left(1 / \tau_{k}\right)}\left(\left|f\left(t_{i}^{k}\right)-f\left(t_{i-1}^{k}\right)\right| / \sqrt{T_{i}}+\sqrt{\lambda \log \left(1 / \tau_{k}\right)}\right)}
\end{aligned}
$$




$$
\begin{aligned}
& \geqslant \frac{1}{\sqrt{\lambda \log \left(1 / \tau_{k}\right)}\left(2 \sqrt{\log (n)}+\sqrt{\lambda \log \left(1 / \tau_{k}\right)}\right)} \quad \text { since } f \in F_{n} \\
& \geqslant \frac{1}{\sqrt{\lambda \log \left(1 / \tau_{k}\right)}\left(2 \sqrt{\log \left(1 / \tau_{n}\right)}+\sqrt{\lambda \log \left(1 / \tau_{k}\right)}\right)} \quad \text { since } \tau_{n} \leqslant 1 / n \\
& \geqslant \frac{1}{\sqrt{\lambda \log \left(1 / \tau_{n}\right)}\left(2 \sqrt{\log \left(1 / \tau_{n}\right)}+\sqrt{\lambda \log \left(1 / \tau_{n}\right)}\right)} \quad \text { since } \tau_{n} \leqslant \tau_{k} \\
& =\frac{1}{\log \left(1 / \tau_{n}\right)(\lambda+2 \sqrt{\lambda})} .
\end{aligned}
$$

\section{Lemma 9.}

$$
P\left(\sum_{i=1}^{n} \rho_{i}^{n} \geqslant \frac{n}{8(\lambda+2 \sqrt{\lambda})(2+2 \sqrt{A(\lambda)})^{2} \log \left(1 / \tau_{n}\right)}\right) \rightarrow 1 .
$$

Proof. Let $f \in F_{n}$. Then for any $k \leqslant n$,

$$
\rho^{k} \geqslant \frac{1}{\log \left(1 / \tau_{n}\right)(\lambda+2 \sqrt{\lambda})}
$$

by Lemma 8 . Suppose that subinterval $\left[t_{i-1}^{n}, t_{i}^{n}\right]$ was produced at time $k_{i}<n$; say $\rho^{k_{i}}=$ $\rho_{j}^{k_{i}}, t_{k_{i}+1}=\left(t_{j-1}^{k_{i}}+t_{j}^{k_{i}}\right) / 2$, and $f\left(t_{j-1}^{k_{i}}\right) \geqslant f\left(t_{j}^{k_{i}}\right)$. Let $m=m(n)=\min _{1 \leqslant i \leqslant n} k_{i}$, and observe that $m(n) \rightarrow \infty$ as $n \rightarrow \infty$ since the observations become dense. Assume that $f \in F_{m} \cap F_{n}$. Since $m=m(n) \rightarrow \infty, P\left(F_{m} \cap F_{n}\right) \rightarrow 1$ as $n \rightarrow \infty$.

Since $f \in F_{n}$,

$$
\rho_{j}^{k_{i}}=\rho^{k_{i}} \geqslant \frac{1}{\log \left(1 / \tau_{n}\right)(\lambda+2 \sqrt{\lambda})} .
$$

Since we assume that $f\left(t_{j-1}^{k_{i}}\right) \geqslant f\left(t_{j}^{k_{i}}\right)$, the smaller child of the split will have

$$
\begin{aligned}
\rho_{j}^{k_{i}+1}= & \frac{1}{2} \frac{t_{j}^{k_{i}}-t_{j-1}^{k_{i}}}{\left(f\left(t_{j-1}^{k_{i}}\right)-M_{k_{i}+1}+g\left(\tau_{k_{i}+1}\right)\right)\left(f\left(t_{k_{i}+1}\right)-M_{k_{i}+1}+g\left(\tau_{k_{i}+1}\right)\right)} \\
= & \frac{1}{2} \rho^{k_{i}} \frac{\left(f\left(t_{j-1}^{k_{i}}\right)-M_{k_{i}}+g\left(\tau_{k_{i}}\right)\right)\left(f\left(t_{j}^{k_{i}}\right)-M_{k_{i}}+g\left(\tau_{k_{i}}\right)\right)}{\left(f\left(t_{j-1}^{k_{i}}\right)-M_{k_{i}+1}+g\left(\tau_{k_{i}+1}\right)\right)\left(f\left(t_{k_{i}+1}\right)-M_{k_{i}+1}+g\left(\tau_{k_{i}+1}\right)\right)} \\
\geqslant & \frac{1}{2} \rho^{k_{i}} \frac{\left(f\left(t_{j-1}^{k_{i}}\right)-M_{k_{i}}+g\left(\tau_{k_{i}}\right)\right)\left(f\left(t_{j}^{k_{i}}\right)-M_{k_{i}}+g\left(\tau_{k_{i}}\right)\right)}{\left(f\left(t_{j-1}^{k_{i}}\right)-M_{k_{i}+1}+g\left(\tau_{k_{i}}\right)\right)\left(f\left(t_{k_{i}+1}\right)-M_{k_{i}+1}+g\left(\tau_{k_{i}}\right)\right)} \\
= & \frac{1}{2} \rho^{k_{i}} \frac{\frac{f\left(t_{j-1}^{k_{i}}\right)-M_{k_{i}}+g\left(\tau_{k_{i}}\right)}{f\left(t_{j}^{k_{i}}\right)-M_{k_{i}}+g\left(\tau_{k_{i}}\right)}}{\left(\frac{f\left(t_{j-1}^{k_{i}}\right)-M_{k_{i}}+g\left(\tau_{k_{i}}\right)}{f\left(t_{j}^{k_{i}}\right)-M_{k_{i}}+g\left(\tau_{k_{i}}\right)}+\frac{M_{k_{i}}-M_{k_{i}+1}}{f\left(t_{j}^{k_{i}}\right)-M_{k_{i}}+g\left(\tau_{k_{i}}\right)}\right)} \\
& \times \frac{1}{\left(\frac{f\left(t_{k_{i}+1}\right)-M_{k_{i}}+g\left(\tau_{k_{i}}\right)}{f\left(t_{j}^{k_{i}}\right)-M_{k_{i}}+g\left(\tau_{k_{i}}\right)}+\frac{M_{k_{i}}-M_{k_{i}+1}}{f\left(t_{j}^{k_{i}}\right)-M_{k_{i}}+g\left(\tau_{k_{i}}\right)}\right)}
\end{aligned}
$$




$$
\geqslant \frac{1}{2} \rho^{k_{i}} \frac{\frac{f\left(t_{j-1}^{k_{i}}\right)-M_{k_{i}}+g\left(\tau_{k_{i}}\right)}{f\left(t_{j}^{k_{i}}\right)-M_{k_{i}}+g\left(\tau_{k_{i}}\right)}}{\left(\frac{f\left(t_{j-1}^{k_{i}}\right)-M_{k_{i}}+g\left(\tau_{k_{i}}\right)}{f\left(t_{j}^{k_{i}}\right)-M_{k_{i}}+g\left(\tau_{k_{i}}\right)}+\frac{M_{k_{i}}-M_{k_{i}+1}}{f\left(t_{j}^{k_{i}}\right)-M_{k_{i}}+g\left(\tau_{k_{i}}\right)}\right)^{2}},
$$

since $f\left(t_{j-1}^{k_{i}}\right) \geqslant f(\bar{t})$. Next observe that since $f \in F_{n}$ and $f\left(t_{j-1}^{k_{i}}\right) \geqslant f\left(t_{j}^{k_{i}}\right)$,

$$
\begin{aligned}
1 & \leqslant \frac{f\left(t_{j-1}^{k_{i}}\right)-M_{k_{i}}+g\left(\tau_{k_{i}}\right)}{f\left(t_{j}^{k_{i}}\right)-M_{k_{i}}+g\left(\tau_{k_{i}}\right)}=1+\frac{f\left(t_{j-1}^{k_{i}}\right)-f\left(t_{j}^{k_{i}}\right)}{\sqrt{t_{j}^{k_{i}}-t_{j-1}^{k_{i}}}} \frac{\sqrt{t_{j}^{k_{i}}-t_{j-1}^{k_{i}}}}{f\left(t_{j}^{k_{i}}\right)-M_{k_{i}}+g\left(\tau_{k_{i}}\right)} \\
& \leqslant 1+2 \sqrt{\log (m)} \frac{\sqrt{A(\lambda)}}{\sqrt{\log (m)}} \quad \text { by Lemma } 5 \\
& =1+2 \sqrt{A(\lambda)} .
\end{aligned}
$$

Next suppose that $M_{k_{i}+1}-M \leqslant g\left(\tau_{k_{i}+1}\right)$. Then

$$
\frac{M_{k_{i}}-M_{k_{i}+1}}{f\left(t_{j}^{k_{i}}\right)-M_{k_{i}}+g\left(\tau_{k_{i}}\right)} \leqslant 1
$$

and so

$$
\rho_{j}^{k_{i}+1} \geqslant \frac{1}{2} \rho^{k_{i}} \frac{1}{(2+2 \sqrt{A(\lambda)})^{2}},
$$

and

$$
\begin{aligned}
\rho_{i}^{n}= & \frac{t_{i}^{n}-t_{i-1}^{n}}{\left(f\left(t_{i-1}^{n}\right)-M_{n}+g\left(\tau_{n}\right)\right)\left(f\left(t_{i}^{n}\right)-M_{n}+g\left(\tau_{n}\right)\right)} \\
\geqslant & \frac{t_{i}^{n}-t_{i-1}^{n}}{\left(f\left(t_{i-1}^{n}\right)-M_{n}+g\left(\tau_{k_{i}+1}\right)\right)\left(f\left(t_{i}^{n}\right)-M_{n}+g\left(\tau_{k_{i}+1}\right)\right)} \\
\geqslant & \rho_{j}^{k_{i}+1}\left(\frac{f\left(t_{i-1}^{n}\right)-M_{k_{i}+1}+g\left(\tau_{k_{i}+1}\right)}{f\left(t_{i-1}^{n}\right)-M_{k_{i}+1}+g\left(\tau_{k_{i}+1}\right)+\left(M_{k_{i}+1}-M\right)}\right) \\
& \times\left(\frac{f\left(t_{i}^{n}\right)-M_{k_{i}+1}+g\left(\tau_{k_{i}+1}\right)}{f\left(t_{i}^{n}\right)-M_{k_{i}+1}+g\left(\tau_{k_{i}+1}\right)+\left(M_{k_{i}+1}-M\right)}\right) \\
= & \rho_{j}^{k_{i}+1}\left(\frac{1}{1+\frac{1}{f\left(t_{i-1}^{n}\right)-M_{k_{i}+1}+g\left(\tau_{k_{i}+1}\right)}}\right)\left(\frac{1}{1+\frac{1}{f\left(t_{i}^{n}\right)-M_{k_{i}+1}+g\left(\tau_{k_{i}+1}\right)}}\right) \\
\geqslant & \rho_{j}^{k_{i}+1}\left(\frac{1}{1+\frac{M_{k_{i}+1}-M}{g\left(\tau_{k_{i}+1}\right)}}\right)\left(\frac{1}{1+\frac{M_{k_{i}+1}-M}{g\left(\tau_{k_{i}+1}\right)}}\right) \\
\geqslant & \frac{1}{4} \rho_{j}^{k_{i}+1} \\
\geqslant & \frac{1}{8(2+2 \sqrt{A(\lambda)})^{2}} \rho^{k_{i}} .
\end{aligned}
$$


Since $f \in F_{n}$, Lemma 2 implies that

$$
P\left(\bigcup_{k=m}^{n}\left\{f: \frac{M_{k}-M}{g\left(\tau_{k}\right)}>1\right\}\right) \leqslant \sum_{k=m}^{n} P\left(\left\{f: \frac{M_{k}-M}{g\left(\tau_{k}\right)}>1\right\}\right) \leqslant \sum_{k=m}^{n} \frac{1}{k^{2 \lambda-1}}
$$

If $\lambda \geqslant 2$, then

$$
\sum_{k=m}^{n} \frac{1}{k^{2 \lambda-1}} \leqslant \sum_{k=m}^{\infty} \frac{1}{k^{3}} \rightarrow 0
$$

as $m \rightarrow \infty$. Therefore,

$$
P\left(\bigcap_{k=m}^{n}\left\{f: \frac{M_{k}-M}{g\left(\tau_{k}\right)} \leqslant 1\right\}\right) \rightarrow 1
$$

On the set

$$
F_{m} \cap F_{n} \cap\left(\bigcap_{k=m}^{n}\left\{\frac{M_{k}-M}{g\left(\tau_{k}\right)} \leqslant 1\right\}\right)
$$

we have

$$
\begin{aligned}
\sum_{i=1}^{n} \rho_{i}^{n} & \geqslant \frac{1}{4} \sum_{i=1}^{n} \rho_{i}^{k_{i}+1} \geqslant \frac{1}{4} \sum_{i=1}^{n} \frac{1}{2} \rho_{i}^{k_{i}} \frac{1}{(2+2 \sqrt{A(\lambda)})^{2}} \\
& \geqslant \frac{1}{8(2+2 \sqrt{A(\lambda)})^{2}} \frac{n}{\log \left(1 / \tau_{n}\right)(\lambda+2 \sqrt{\lambda})}
\end{aligned}
$$

We have shown that with probability approaching 1 ,

$$
\frac{n}{8(2+2 \sqrt{A(\lambda)})^{2} \log \left(1 / \tau_{n}\right)(\lambda+2 \sqrt{\lambda})} \leqslant \sum_{i=1}^{n} \rho_{i}^{n} \leqslant 5 \log \left(1 / g\left(\tau_{n}\right)\right)
$$

which implies that

$$
\begin{aligned}
\frac{n}{c(\lambda)} & \leqslant \log \left(1 / \tau_{n}\right) \cdot \log \left(1 / g\left(\tau_{n}\right)\right) \\
& =\frac{1}{2} \log \left(1 / \tau_{n}\right) \cdot\left(\log \left(1 / \tau_{n}\right)-\log \log \left(1 / \tau_{n}\right)\right),
\end{aligned}
$$

where

$$
c(\lambda) \equiv 40(2+2 \sqrt{A(\lambda)})^{2}(\lambda+2 \sqrt{\lambda}) .
$$


Set $x_{n}=\log \left(1 / \tau_{n}\right)$; then the previous inequality reads

$$
\frac{2 n}{c(\lambda)} \leqslant x_{n}^{2}-x_{n} \log \left(x_{n}\right)
$$

Recall that $x_{n} \rightarrow \infty$. Then $x_{n} \geqslant \sqrt{2 n / c(\lambda)}$, or $\log \left(1 / \tau_{n}\right) \geqslant \sqrt{2 n / c(\lambda)}$, and so

$$
g\left(\tau_{n}\right)=\sqrt{\tau_{n} \log \left(1 / \tau_{n}\right)} \leqslant(\exp (-\sqrt{2 n / c(\lambda)}) \sqrt{2 n / c(\lambda)})^{1 / 2}
$$

By Lemma $2 P\left(\Delta_{n} \leqslant g\left(\tau_{n}\right)\right) \rightarrow 1$, and so

$$
P\left(\Delta_{n} \leqslant(2 n / c(\lambda))^{1 / 4} \exp \left(-\frac{1}{2} \sqrt{2 n / c(\lambda)}\right)\right) \rightarrow 1
$$

This completes the proof of Theorem 1.

Acknowledgement. This material is based upon work supported by the National Science Foundation under Grant No. CMMI-0825381.

\section{References}

Bartkute-Norkuniene, V. (2009). Stochastic optimization algorithms for support vector machines classification. Informatica, 20, 173-186.

Kushner, H. (1962). A versatile stochastic model of a function of unknown and time-varying form. Journal of Mathematical Analysis and Applications, 5, 150-167.

Lifshits, M.A. (1995). Gaussian Random Functions. Kluwer Academic, Dordrecht.

Mockus, J. (1972). On Bayesian methods of search for the extremum. Automatics and Computers, 3, 53-62.

Shepp, L.A. (1979). The joint density of the maximum and its location for a Wiener process with drift. Journal of Applied Probability, 31, 423-427.

Žilinskas, A. (1985). Axiomatic characterization of global optimization algorithm and investigation of its search strategy. OR Letters, 4, 35-39.

J. Calvin is a professor in the Department of Computer Science at the New Jersey Institute of Technology. His research interests are in global optimization, probabilistic analysis of algorithms, and simulation methods.

\section{Adaptyvus vienmatis globalios optimizacijos algoritmas ir jo konvergavimo greitis Vinerio mato atveju}

\section{James CALVIN}

Aprašytas adaptyvus algoritmas skirtas aproksimuoti vieno kintamojo funkcijos globaluji minimumą. Paklaidos konvergavimo greitis yra įvertintas darant prielaidą, kad tikslo funkcijos atsitiktinès pasiskirsčiusios pagal Vinerio matą. 